\title{
Experience gained with the 3D machining of the W7-X HHF divertor target elements
}

\author{
P. Junghanns ${ }^{\mathrm{a}}$, J. Boscary ${ }^{\mathrm{b}}$, A. Peacock ${ }^{\mathrm{b}}$ \\ ${ }^{a}$ Max Planck Institute for Plasma physics, Greifswald, Germany \\ ${ }^{b}$ Max Planck Institute for Plasma physics, Garching, Germany
}

The high heat flux (HHF) divertor of W7-X consists of 100 target modules assembled from 890 actively water-cooled target elements protected with CFC tiles. The divertor surface will be built up of individually 3D machined target elements with 89 individual element types. To date 300 of the 890 target elements have been 3D machined with a very good accuracy. To achieve this successful result, a prototyping phase has been conducted to qualify the manufacturing route and to define the acceptance criteria with measures taken to minimize the risk of unacceptable damage during the manufacturing. After the 3D-machining, during the incoming inspection, copper infiltration from the interface between the CFC tiles and the CuCrZr heat sink to the plasma facing surface was detected in a small number of elements.

Keywords: Stellarator, Wendelstein 7-X, In-Vessel Components, Divertor, Target Element, CFC, 3D-machining.

\section{Introduction}

The plasma facing components [1] of the W7-X machine are actively-cooled to remove an input power of $10 \mathrm{MW}$ for plasma pulse lengths of up to 30 minutes and peak power with additional combined heating systems of up to $24 \mathrm{MW}$ for $10 \mathrm{~s}$ [2]. These components are mostly covered with carbon-based materials and have surfaces facing the plasma with an area of approximately $265 \mathrm{~m}^{2}$. For a large part of these surfaces significant care is taken, in the design, manufacture and assembly of the components to avoid leading edges which could be exposed to high power loads from plasma particles traveling along field lines and intersecting with leading edges with the consequence of local overheating and material sublimation. This issue is particularly important for the high heat flux (HHF) divertor targets which receive the highest thermal loads.

The divertor target has a complex $19 \mathrm{~m}^{2} 3-\mathrm{D}$ shape but the target modules are made of components of finite width, i.e. the target elements. The technology for the elements has been optimized [3] to obtain elements which are as wide as possible; from $50 \mathrm{~mm}$ up to $61.5 \mathrm{~mm}$ wide depending on the position in the divertor. This is significantly wider than monoblock [4] technology but still there are a significant number of potential edges.

The divertor is made of 10 similar discrete units and each unit has 10 different modules. The 100 modules of the HHF divertor [5] are constructed from 890 individual elements of five main types attached onto a support frame and welded together to water manifolds. The 3-D surface of the module is formed by $3 \mathrm{D}$ machining the elements individually before they are installed on the module. This manufacturing route was chosen because it was easier to clean the individual elements rather than the complicated modules and the risk of damaging an edge during the machining of a module was considered to be higher and also the consequences of a damaged edge in a machined module is also greater; the module needing to be dismantled to exchange the damaged element.

Nevertheless the machining of the 3D surface of the individual elements with the friable CFC [6] surface material with minimum damage or chipping of the edges required significant development to find the appropriate technology, qualify the process, identify the best manufacturing route, control the selected process and define the acceptance criteria for the elements. The results of the manufacture for the first set of 300 target elements are presented.

3-D machining flaws in the CFC material also needed to be assessed to determine whether or not they could have consequences on the performance of the elements. Results of this assessment are also shown.

\section{Module and element design}

\subsection{Module design}

Fig. 1 shows the design of TM8h. The module consists of 12 elements mounted on a frame with a gap of typically $1 \mathrm{~mm}$ between neighbouring elements.

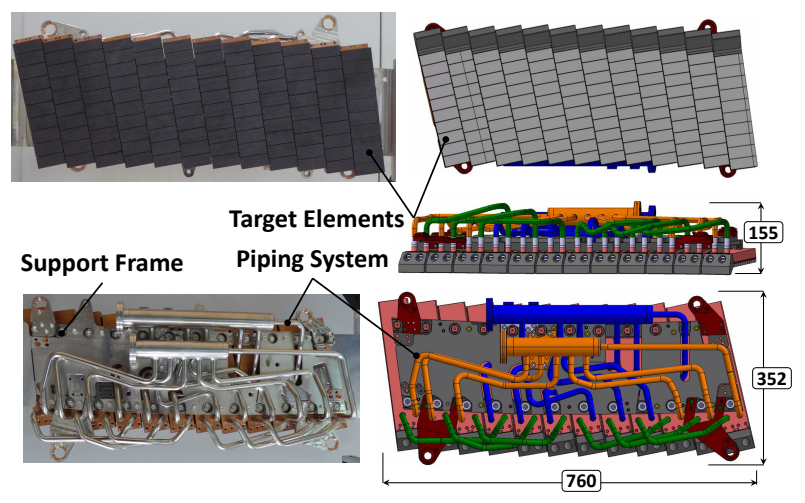

Figure 1: Design of a target module TM8h (dry weight: $55 \mathrm{~kg}$ ) 
The contour tolerance specified for the surface of the stainless steel frame onto which the target elements are positioned is $\pm 0.25 \mathrm{~mm}$. The measurements confirmed that the frames have been manufactured within this tolerance.

\subsection{Element design}

The 300 elements are built into the target modules (TMs) TM7h, TM8h and TM9h. There are 230 type 4 elements and 60 type 3 elements with a length of 250 and $320 \mathrm{~mm}$, respectively. Fig. 2 shows the design of a type 4 element installed in TM8h.

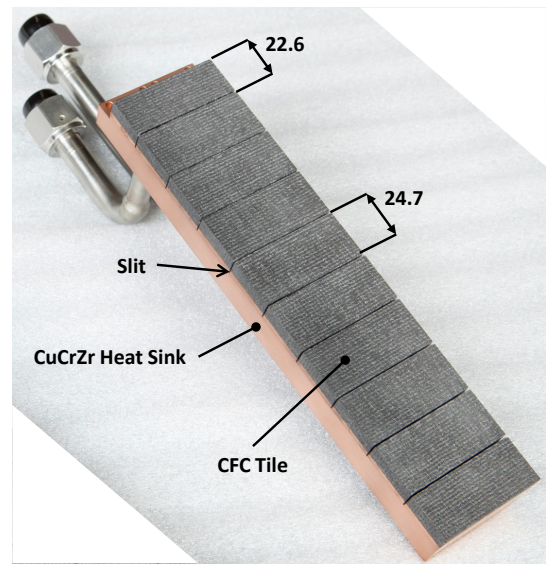

Figure 2: Design of the target element of type 4

This element has 10 tiles with a slit of $0.8 \pm 0.2 \mathrm{~mm}$ between tiles. The unique 3D form of each element depends on its position in the module. Since the divertor consists of 10 similar units, there are only 10 elements of each type. The attachment of the element to the frame is performed with two location studs. Around the studs is an area with a guaranteed flatness tolerance, which corresponds to the contact area of the element to the module frame. The 3-D surface has been defined in such a way as to minimize the amount of material that has to be removed and maximize the remaining material. The initial CFC tile thickness before machining is $8 \mathrm{~mm}$. However, one of the unclear points is the minimum amount of material that could be removed, which depends upon the technique used. As part of the qualification it was necessary to find this out.

\section{Prototype production}

Experience has been gained previously with graphite machining at IPP for the tiles of inertially cooled divertor, which will be operate before the activelycooled divertor [7]. This used a combination of machining of the back surface with larger tools and the machining of the front side with ball nosed cutters. The technique was also tried with the CFC surface. Initially small pieces were machined to ensure the correct cutting speed and to ensure that no breaking of the surface was seen. These tests were used to establish the cutting speed as well as the cutting direction and it was found that the direction of the cutter as it entered the test piece was important to ensure no break out of the material. In addition the likelihood of damage occurring is at the highest at the edges of the element. In order to minimize machining time another strategy developed was to concentrate the machining efforts on the edges of the tiles and machine the central region with a lower quality. A schematic of this machining strategy is shown in Fig.3.

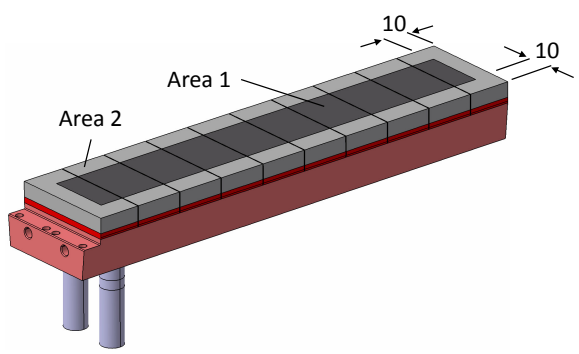

Figure 3: Machining strategy

In order to further qualify the machining process six pre-series elements of type 4 were 3 -D machined and mounted on a prototype module (Fig. 4, right side). This prototype module was then completely built together and tested in Gladis [8]. The different loaded areas during the HHF testing are visible on the CFC surface of the module.

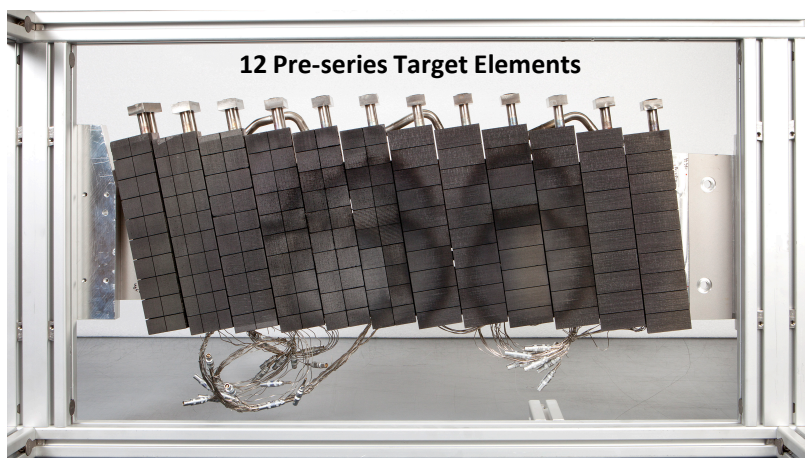

Figure 4: Prototype of target module (TM9h)

The machining process for the CFC is dry and it was important to establish that under the high heat loading there was no surface contamination and no spitting of the surface as had been seen with other types of machining. The Gladis tests showed clean surface with no evidence of surface contamination. In Gladis, the beam is perpendicular to the loaded surface; the leading edge effects cannot be simulated. The steps between neighbouring elements were $3 \mathrm{D}$ measured and showed a very good agreement with the CAD model.

\section{Optimisation for series element machining}

\subsection{Development of acceptance criteria}

Previous experience with the handling of CFC and its machining had showed that it would never be possible to achieve perfect edges. Consideration was then given to what would be an acceptable level of damage. In order to do this, calculations were performed with idealized defects on the surface of the elements. Taking different configurations into account and looking at the worst case scenario of full convective load then it was possible to determine different damage cases that would be acceptable. Fig.5 shows a typical example of the damage that could be allowed, for example, on the surface of the tile. These different cases were used as 
the basis of the technical specification sent to the firms given the task of producing the elements and for an assessment of the basis of the quality of the machined elements.

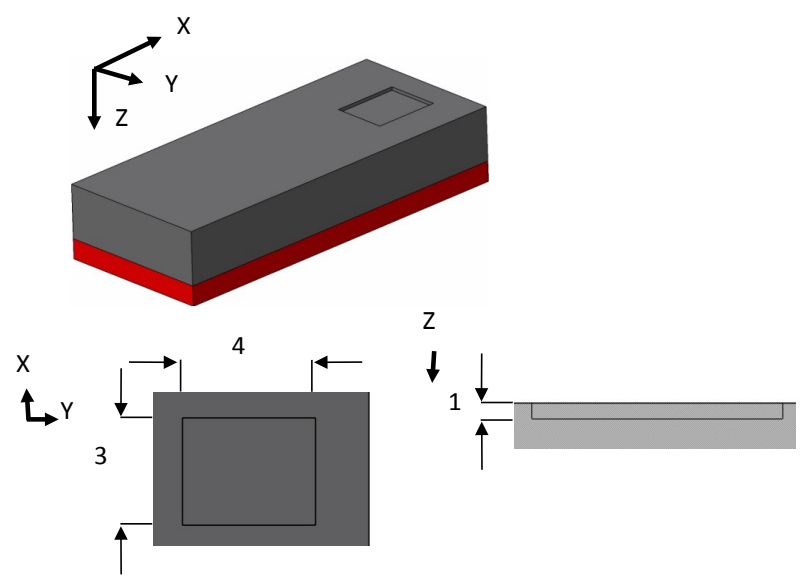

Figure 5: typical allowed damage of a target element tile

\section{Series element machining}

Based on the results of the prototype elements, the developed acceptance criteria and inspection methods a number of firms were asked to provide offers for the 3-D machining of the 300 elements of types 4 and 3. After a call for tender with the evaluation of manufactured test pieces, the selected company was the firm Gewo $\mathrm{GmbH}$ (Germany). The machining process and further details presented are based on the agreed programme of work with this firm.

\subsection{Clamping jigs and reference systems}

In order to achieve the highest accuracy of machining a jig was developed by the company which replicated the attachment features of the module frame. In this way the element was supported in the same way that it is on the module.

\subsection{Machining}

Prior to the machining of each element type (10 per series) a graphite test piece of the same geometry was machined and measured to ensure the correct programme was being used and that there was no problems with the machining system (Fig. 6).

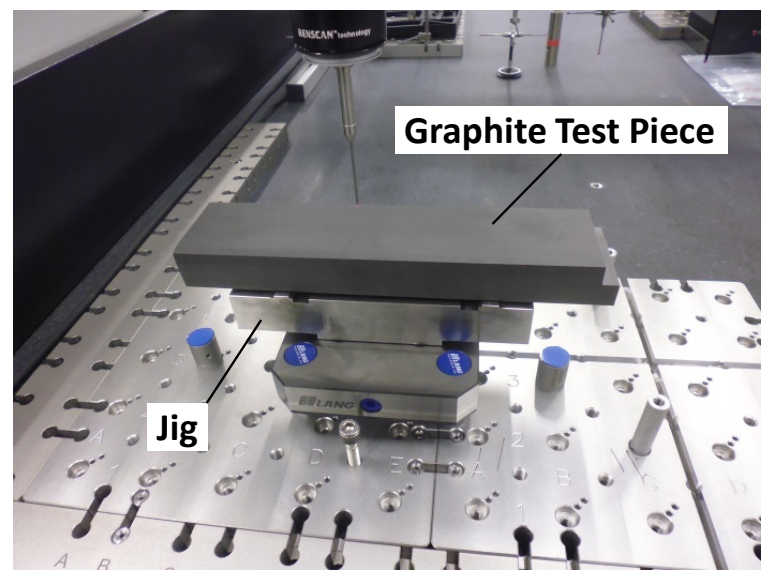

Figure 6: Measurement of graphite test piece (Courtesy of Gewo GmbH)
After this the target elements were machined in a 5 axis milling machine especially adapted for the machining of CFC (Fig.7).

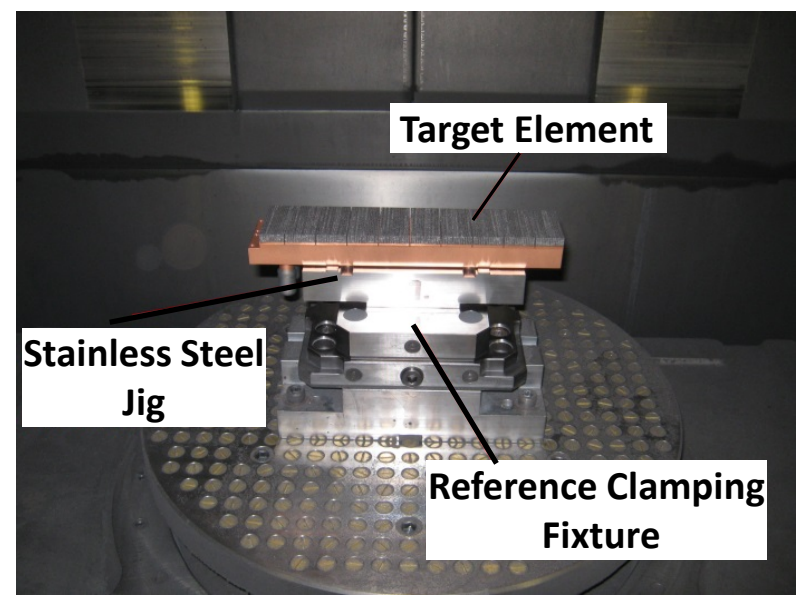

Figure 7: Target element to be machined (Courtesy of Gewo $\mathrm{GmbH}$ )

\subsection{Inspection}

After the machining the elements are immediately measured positioned on the jig by the company. The maximal allowable surface roughness is $\mathrm{Ra}=6.4 \mu \mathrm{m}$. The specified contour tolerance of the CFC surface is $\pm 0.025 \mathrm{~mm}$. Results in Table 1 show an example of the accuracy achieved with the machining of the elements. The measurement points are indicated in Fig. 8.

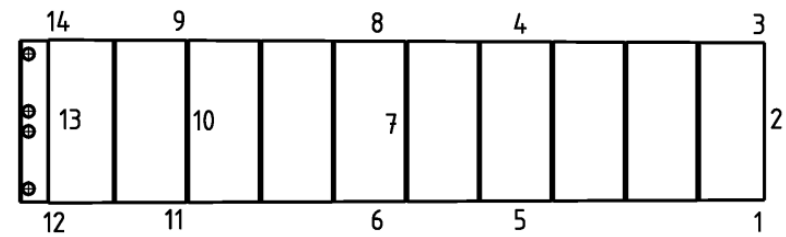

Figure 8: Location of the measurement points for type 4

\begin{tabular}{|r|c|c|c|}
\hline & Measured value & Specified value & Difference \\
\hline 1 & 29.195 & 29.187 & 0.008 \\
\hline 2 & 29.088 & 29.087 & 0.001 \\
\hline 3 & 29.000 & 29.005 & -0.005 \\
\hline 4 & 29.061 & 29.064 & -0.003 \\
\hline 5 & 29.125 & 29.121 & 0.004 \\
\hline 6 & 29.091 & 29.084 & 0.007 \\
\hline 7 & 29.085 & 29.084 & 0.001 \\
\hline 8 & 29.096 & 29.100 & -0.004 \\
\hline 9 & 29.150 & 29.152 & -0.002 \\
\hline 10 & 29.086 & 29.085 & 0.001 \\
\hline 11 & 29.039 & 29.035 & 0.004 \\
\hline 12 & 29.008 & 29.007 & 0.001 \\
\hline 13 & 29.086 & 29.088 & -0.002 \\
\hline 14 & 29.179 & 29.184 & -0.005 \\
\hline
\end{tabular}

Table 1: Results of measurement in $\mathrm{mm}$ for elements in comparison to their theoretical value

The analysis of the measured values of the 300 elements showed an achieved tolerance for all of the elements of $<$ $\pm 0.015 \mathrm{~mm}$. 
All the elements were visually inspected after machining for signs of damage and then carefully packed into special carrying cases for transport back to IPP

\section{Incoming inspections}

As well as being inspected at the company the elements were all fully inspected on arrival at IPP. This was to ensure that there was no transport damage and no elements had been incorrectly machined. Some elements were randomly selected and also re-measured to ensure that there were no systematic problems with the machining in the company.

All elements were correctly machined and the machining was performed $100 \%$ correctly. However, during the incoming inspections small spots of copper were seen on the CFC surfaces. An example is shown in Fig.9.

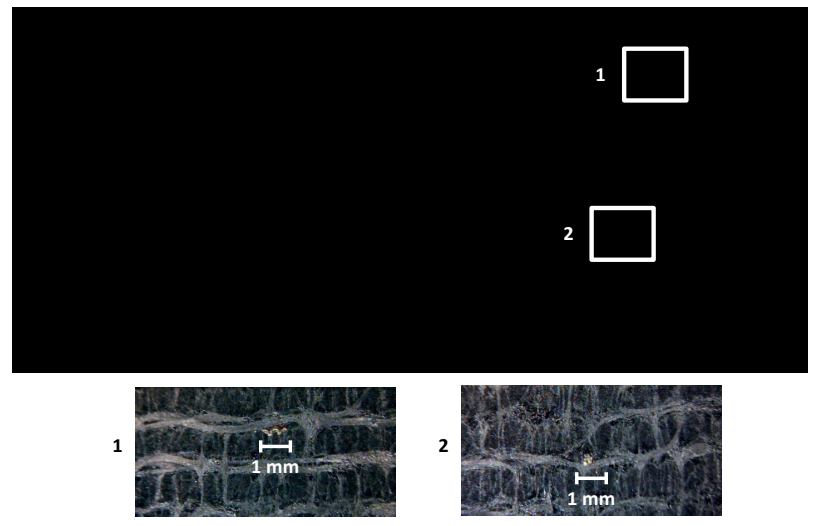

Figure 9: First examples seen of copper in the tile surface

A systematic investigation was then performed on all of the target elements. The 300 target elements have 3180 tiles and 51 tiles (1.6\%) have shown copper on the surface. The total surface of the 300 elements is $4.43 \mathrm{~m}^{2}$ and the total estimated copper surface is $<3 \mathrm{~mm}^{2}$.

The reason for the copper in the tiles was assumed to be due to the presence of copper used to bind the tiles to the cooling structure by active metal casting. This was investigated in these particular examples with the help of X-ray picture (Fig. 10).

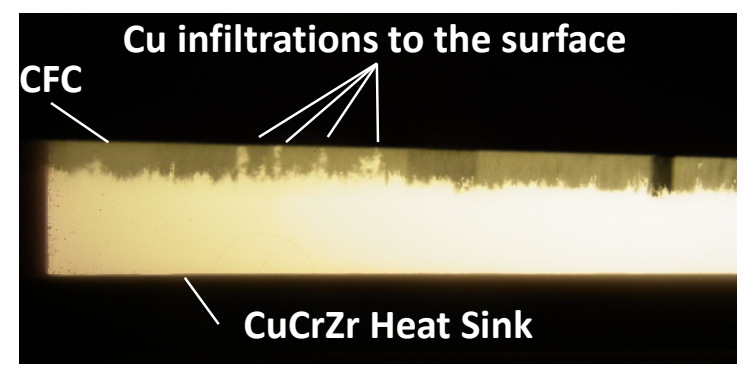

Figure 10: $\mathrm{X}$ ray picture of copper infiltration

The visual inspections correlated with the X-ray pictures. There are holes in the complicated CFC structure of the tile and sometimes copper found a way to reach the surface.

\section{Conclusions}

The machining of the 3-D surface of the HHF divertor target elements was considered to be one of the many challenging aspects of the production of the divertor. The high accuracy requirements and the friable nature of the CFC necessitated a detailed work programme to qualify the necessary technology and a clear definition of the manufacturing controls and inspections. The planned work programme has been brought to a conclusion for the first 300 delivered elements. An unexpected occurrence of $\mathrm{Cu}$ on the surface of the elements has also been discovered. This copper appears to come from the copper used to infiltrate the CFC. Assessments show that the amount of copper on the surface is similar to the native impurity level in the CFC and should be no risk to operation. Nevertheless the elements which show the presence of copper have all been grouped together in the same module so that experiments with impurities could be attempted at specific locations. The technology is in place for the 3-D machining and the machining of the 590 remaining target elements is planned in the near future.

\section{Acknowledgments}

This project has received funding from the European Union's Horizon 2020 research and innovation programme under grant agreement number 633053. The views and opinions expressed herein do not necessarily reflect those of the European Commission.

\section{References}

[1] R. Stadler et al.,The in-vessel components of the experiment W 7-X, Fus. Eng. and Des. 84 (2009) 305308.

[2] M. Gasparotto et al., Wendelstein 7-X - Status of the project and commissioning planning, Fus. Eng. and Des. 89 (2014) 2121-2127.

[3] J. Boscary et al., Optimisation of target plates for the W7$\mathrm{X}$ divertor at stationary operation, Fus. Eng. and Des. 5657 (2001) 279-283.

[4] S Carpentier-Chouchana, et al., Status of the ITER fulltungsten divertor shaping and heat load distribution analysis, Phys. Scr. T159 (2014).

[5] J. Boscary et al., Water-cooled target modules for steadystate operation of the W7-X divertor, Nucl. Fusion 43 (2003) 831-834.

[6] A. T. Peacock et al., Status of CFC development in Europe for ITER, Phys. Scr. T128 (2007) 23-28.

[7] A. Peacock et al., Progress in the design and development of a test divertor (TDU) for the start of W7-X operation, Fus. Eng. and Des. 84 (2009) 1475-1478.

[8] A. Peacock et al., Status of High Heat Flux Components at W7-X, IEEE Trans. Plasma Science 42 (3) (2104) 524532. 